\title{
Identification and analysis of the expression of microRNA from lactating and nonlactating mammary glands of the Chinese swamp buffalo
}

\author{
Xiaoyan Cai, ${ }^{*}$ † Qingyou Liu, ${ }^{* 1}$ Xiaoxi Zhang, ${ }^{*}$ Yanping Ren, ${ }^{*}$ Xiaocan Lei, ${ }^{*}$ Sheng Li, ${ }^{*}$ Qiuping Chen, \\ Kai Deng, ${ }^{*}$ Ping Wang, ${ }^{*}$ Haihang Zhang, ${ }^{*}$ and Deshun Shi*1 \\ *State Key Laboratory for Conservation and Utilization of Agricultural Bioresources in the Subtropics, Guangxi University, Nanning, China 530004 \\ †Guangxi Institute of Animal Science, Nanning, China, 530001
}

\begin{abstract}
To study the role of microRNA (miR) in the lactation physiology of water buffalo, 2 multiparous dairy buffaloes (including an 8-yr-old buffalo that had been lactating for $3 \mathrm{mo}$, as well as a 10-yr-old nonlactating, nonpregnant buffalo) were used for miR library construction. The profile of differentially expressed miR in lactating and nonlactating mammary gland tissues of these water buffalo were investigated using Illumina-Solexa high-throughput sequencing technology (Illumina, San Diego, CA). The data identified $259 \mathrm{miR}$ families, 359 mature miR, 363 pre-miR, 230 novel buffalo $\mathrm{miR}$, and 5 buffalo-specific miR that were expressed in mammary tissues. Some highly significantly differentially expressed miR were explored, including bbu-miR-497, bbu-miR-30a-5p, bbu-miR148a, bbu-miR-29a, bbu-miR-125a, bbu-miR-125b, and bbu-miR-103. The expression patterns of $18 \mathrm{miR}$ were confirmed by quantitative real-time PCR in both tissues, and the expression of bbu miR-103 and novel miR-57 constituted the largest differences between lactating and nonlactating tissues. Further functional analysis indicated that the overexpression or suppression of miR-103 in buffalo mammary epithelial cells downregulated or upregulated the expression of pantothenate kinase 3 , and also significantly increased the transcription factor steroid regulatory element binding protein, followed by the acceleration of de novo synthesis of fatty acids by upregulation of acetyl coenzyme A carboxylase $\alpha$ expression. The expression levels of 34 predicted target genes of novel-miR-57 in lactating and nonlactating mammary gland tissues were all analyzed by quantitative real-time PCR. Finally, only the expression of docking protein 4 could be upregulated or downregulated selectively by bbu-novel-miR-57
\end{abstract}

Received May 15, 2016

Accepted November 20, 2016.

${ }^{1}$ Corresponding authors: qyliu2002@gmail.com and ardsshi@gxu. edu.cn in buffalo mammary epithelial cells and the Bcap-37 cell line. This study provides an overview of the miR expression profile of water buffalo and the interaction between some key miR and their target genes, which may improve understanding of the important roles of miR in buffalo milk fat synthesis.

Key words: buffalo mammary gland, lactation, Solexa high-throughput sequencing, Bbu-miR-103, novelmiR-57

\section{INTRODUCTION}

MicroRNA ( $\mathbf{m i R}$ ) are a group of small [approximately 19-25 nucleotides (nt)], noncoding, and endogenous RNA that regulate protein expression by binding to target mRNA so as to repress translation or promote degradation of the target mRNA (Carrington and Ambros, 2003; Bartel, 2004), or to activate translation at the post-transcriptional level in the RNA-induced silencing complex (Vasudevan et al., 2007). It is estimated that the expression of $30 \%$ of protein-coding genes are regulated by miR (Lewis et al., 2003; Lim et al., 2003). It has been established that miR play important roles in cell differentiation, proliferation, as well as apoptosis in plants, animals, and even viruses (Brennecke et al., 2003; Xie et al., 2011; Miao et al., 2016). Studies of the role of miR in mammary gland biology have mainly focused on the identification of specific miR as tumor suppressors and oncogenes, which have a regulatory effect on gene expression, by targeting mRNA in breast cancer (Le Quesne and Caldas, 2010). During the lactation process, the mammary gland undergoes a cycle of cell proliferation, differentiation, de-differentiation, and death, making it an ideal model organ for investigating the molecular mechanisms underlying mammary gland physiology and lactation (Ji et al., 2012a,b).

Work on the miRNome in human and mouse tissues has shown that, in humans, the expression of $23 \mathrm{miR}$ is specific to breast tissue, whereas, in mice, only $9 \mathrm{miR}$ are specific to mammary tissues (Liu et al., 2004). This suggests that miR play an important role in mammary 
gland physiology. Lactation is the most important function of the mammary glands, and several recent reports have indicated the crucial role played by miR in the regulation of lactation in livestock animals $(\mathrm{Ji}$ et al., 2012b; Li et al., 2012a,c; Wang et al., 2012; Lin et al., 2013; Bu et al., 2015). However, a paucity of information exists regarding the roles of miR in terms of the post-transcriptional regulation of lactation in mammary gland tissues of the Chinese swamp buffalo (Bubalus bubalis).

Water buffalo are important milk-producing animals in the tropical and subtropical regions, contributing to more than $5 \%$ of the global milk supply and 20 to $30 \%$ of Southeast Asia's farm power. Buffalo milk has a higher milk fat ( 8.0 vs. $3.5 \%)$ and protein (4.5 vs. $3.2 \%$ ) content than dairy cattle milk, but a lower milk yield, at 1,200 to 2,000 kg/yr (Shi et al., 2012). For this reason, it is important to understand the gene regulatory networks involved in the lactation physiology of the water buffalo to formulate specialized breeding programs to augment milk production. Although several studies have focused on the role of miR in the lactation physiology of mammary glands of other milk-producing livestock, the miR profile in the lactating and nonlactating mammary gland tissue of the water buffalo is still unknown. Ours is the first report of miR in lactating and nonlactating mammary gland tissues of the water buffalo and contributes significantly to a better understanding of the molecular regulatory mechanisms governing lactation physiology in B. bubalis.

\section{MATERIALS AND METHODS}

\section{Mammary Gland Sample Preparation}

Two multiparous dairy buffaloes were used for miR library construction. One was an 8-yr-old buffalo that had been lactating for 3 mo, used to construct the lactation miR library. The other was a 10 -yr-old nonlactating, non-pregnant buffalo, used to construct the nonlactation miR library. These 2 buffalo were slaughtered according to the Regulations for the Administration of Affairs Concerning Experimental Animals (Ministry of Science and Technology, China, revised in June 2004; http://pkulaw.cn/fulltext_form.aspx?Gid=174603), as well as by approval of the Institutional Animal Care and Use Committee at Guangxi University, China.

Three pieces of mammary gland (technical replicates) tissues were sampled from each buffalo. Both sets of mammary gland samples were immediately frozen in liquid nitrogen and then stored separately at $-80^{\circ} \mathrm{C}$. Total RNA were extracted from the collected mammary gland tissues. Three tubes of peak lactation period total RNA were pooled in equal amounts for Illumina Solexa sequencing (Illumina, San Diego, CA), and the same treatment was performed with the dry period buffalo mammary gland tissues.

\section{RNA Extraction}

Frozen tissue samples were homogenized in Trizol reagent (Ambion, Life Technologies, NY), after which total RNA was extracted from the supernatant according to the manufacturer's protocol (Invitrogen, Carlsbad, CA). The quantity and integrity of RNA were confirmed using a NanoDrop ND-1000 spectrophotometer (NanoDrop Technologies, Wilmington, DE) and Agilent 2100 Bioanalyzer (Agilent Technologies, Santa Clara, CA). Only RNA samples with integrity scores $\geq 7$ were used for further sequencing and analysis of miR, and only RNA samples with an integrity score $\geq 8$ were used for gene (mRNA) sequencing and analysis. Total RNA was stored at $-80^{\circ} \mathrm{C}$ until further use.

\section{Small RNA Library Construction and Solexa Sequencing}

Small RNA ( $<30 \mathrm{nt}$ ) were purified using PAGE and then ligated with a pair of Solexa adaptors to their $3^{\prime}$ and $5^{\prime}$ ends. The small RNA molecules were amplified for 17 cycles using the adaptor primers and the fragments with both small RNA and adaptors (about $90 \mathrm{bp}$ in length) were isolated from agarose gel. The purified molecules were used for cluster generation and sequencing analysis using an Illumina Solexa Sequencer in accordance with the manufacturer's instructions (Glazov et al., 2008; Hafner et al., 2008). The image files generated by the sequencer were processed to produce a digital-quality data. After masking the adaptor sequences, reads contaminated by rRNA, transfer RNA, mRNA, small nuclear RNA, and small nucleolar RNA were excluded. The sequencing quality of the small RNA reads was assessed and the length distribution was calculated. Clean reads were processed for computational analysis and compared using miRBase V. 17.0 (Griffiths-Jones et al., 2008).

Solexa reads were aligned against the Bos taurus genome (Btau 4.0) using SOAP software (Li et al., 2008). Sequences with perfect matches were retained for further analysis. To analyze the RNA secondary structure of the matched Solexa reads, 100 nt of genomic sequences flanking each side of these sequences were extracted, and the secondary structure was predicted using RNAfold-1.99.1.3 (http://hackage.haskell.org/ package/RNAFold-1.99.1.3) and analyzed using the online computational tool MIREAP V. 0.2 (https:// 
sourceforge.net/projects/mireap/; Li et al., 2012a) under default settings. Stem-loop hairpins were considered typical when the sequences met the following criteria: (1) mature miR must be found in 1 arm of the hairpin precursors lacking large internal loops or bulges; (2) the secondary structures of the hairpins must be steady, with free energy of hybridization below $-18 \mathrm{kcal} / \mathrm{mol}$; and (3) hairpins are located in intergenic regions or introns. Finally, the candidate miR reads were used as queries in the BLAST searches (Altschul et al., 1990) of the miRBase database 17.0, with a tolerance level of 2 mismatches.

\section{Prediction of Target Genes of miR}

Each identified miR was located in the genome using the search function in the Ensembl genome browser (http://www.ensembl.org/Bos_taurus); prediction of target genes was performed using MIREAP. The basic rules of prediction for MIREAP were (1) no more than 4 mismatches between small RNA and the target (G-U bases count as 0.5 mismatches); (2) no more than 2 adjacent mismatches in the miR/target duplex; (3) no adjacent mismatches in positions 2 to 12 of the miR/ target duplex; (4) no mismatches in positions 10 to 11 of miR/target duplex; (5) no more than 2.5 mismatches in positions 1 to 12 of the miR/target duplex; and (6) the minimum free energy of the miR/target duplex should be $\geq 75 \%$ of the minimum free energy of the miR bound to its perfect complement. A Blastx search was performed using the target sequence and the Ensembl database (http://asia.ensembl.org/index.html) to predict the functions of potential targets.

\section{Differential Expression Analysis of miR}

To compare the differential miR expression levels between the 2 libraries (lactation and nonlactation), first, the expression of miR in the 2 samples was normalized to determine the expression of transcripts per million. If the normalized expression value of a given miR was normalized to 0 , then its modified expression value would be 0.01 . If the normalized expression value of a given miR was below 1 in both libraries, the miR was removed from future differential expression analysis. The fold-change and $P$-value were calculated from the normalized expression using the following formulas:

\section{Normalized expression $=($ actual $\mathrm{miR}$ sequencing}

reads count/total number of clean reads $) \times 1,000,000$;

Fold change $=\log _{2}($ lactation $/$ nonlactation $) ;$ and
$P$-value:

$$
\begin{gathered}
p(x \mid y)=\left(\frac{L}{N}\right) \frac{(x+y) !}{x ! y !\left(1+\frac{L}{N}\right)^{(x+y+1)},} \\
C\left(y \leq y_{\min } \mid x\right)=\sum_{y=0}^{y \leq y_{\min }} p(y \mid x), \\
D\left(y \geq y_{\max } \mid x\right)=\sum_{y \geq y_{\max }}^{\infty} p(y \mid x) .
\end{gathered}
$$

Here, $N$ and $x$ represent the total number of clean reads and normalized expression levels of a given miR in the nonlactation small RNA library, respectively; $L$ and $y$ represent total number of clean reads and normalized expression levels of a given miR in the lactation small RNA library, respectively; $p$ is the $P$-value for such a table to occur in the hypothesis that actin EST frequencies are independent of cDNA libraries. To compute the confidence intervals, we made use of the $C$ (cumulative) and $D$ (distributions), which allow the computation of an interval $\left[y_{\min }, y_{\max }\right] \varepsilon$ and serve as a significance test when comparing (Audic and Claverie, 1997).

For differentially expressed mRNA detection, we employed IDEG6 (http://telethon.bio.unipd.it/bioinfo/ IDEG6/) to identify miR showing statistically significant differences in relative abundance (as reflected by the total count of individual sequence reads) between the 2 small RNA libraries. We employed the general $\chi^{2}$ method because it resulted in the most efficient tests (Romualdi et al., 2003). Finally, miR were deemed significantly different between the 2 samples when the $P$ $\leq 0.05$ and the ratio between the 2 samples was $>1.5$.

\section{Quantitative Real-Time PCR Assay}

Quantitative real-time (qRT)-PCR was performed using a mirVana miRNA Isolation Kit (Ambion, Thermo Fisher Scientific, Waltham, MA), according to the manufacturer's instructions. Briefly, $5 \mu \mathrm{L}$ of total RNA was reverse-transcribed to cDNA using AMV reverse transcriptase (TaKaRa, Shiga, Japan) and stem-loop RT primers (Ambion); U6 served as the endogenous control for all miR. $\beta$-Actin served as the endogenous control for docking protein 4 (DOK4) and genes involved in the milk fat pathway relating to bbumiR-103. Nematode miR-39 served as the endogenous control for 34 target genes of novel-57. Primers were designed using miR sequences of Chinese swamp buf- 
falo. The qRT-PCR was performed using SYBR Green PCR Master Mix (Applied Biosystems, Foster City, CA) on an Applied Biosystems 7500 Sequence Detection System (Johnson et al., 2013). The threshold cycle values indicated the quantity of the target gene in each sample. Negative controls were performed with water as a template instead of cDNA. Three independent technical or biological samples were used and each run was replicated in triplicate on plates. All related primers were designed using Oligo 6.0 and synthesized by Sangon (Shanghai, China; Supplemental Tables S1-S4, https://doi.org/10.3168/jds.22016-11461).

\section{Hierarchical Clustering of miR Expression}

PermutMatrix software using Pearson distance (http://www.atgc-montpellier.fr/permutmatrix/;

Caraux and Pinloche, 2005) was used to perform hierarchical clustering of $\mathrm{miR}$ expression. The relative cloning frequency for each miR was considered as the number of sequences for each miR in a certain library divided by the total count of sequence reads for this library.

\section{Construction of Lentiviral Vector and Synthesis of Mimics and Inhibitor}

Lentiviral vectors containing the miExpress precursor of miR-103 and a negative control (NC) were constructed by Guangzhou's GeneCopoeia Inc. (Rockville, MD) and referred to as LpEZX-pre-miR-103 and LpEZX-miR-NC. The lentiviral vectors were packaged and propagated in a $293 \mathrm{~T}$ cell line with the packaging plasmid $(\triangle \mathrm{NRF})$ and the envelope plasmid encoding the vesicular stomatitis virus-G glycoprotein (VSVG). The mimics and inhibitor were synthesized by Guangzhou RiboBio Co. Ltd. (Guangzhou, China). The sequence of the miR-103 precursor was cugcccucggcuucuuuacagugcugccuuguugcauauggaucaagcagcaUuguacagggcaugaaggc, whereas that of the miR-103 inhibitor was TCGTCGTUUCUTGTCCCGUTUCT.

\section{Cell Culture}

A $293 \mathrm{~T}$ cell line was maintained in this laboratory and the buffalo mammary epithelial cell (BMEC) was cultured. Briefly, fresh tissue blocks from buffalo during peak lactation were obtained and transported to the laboratory in a standard saline solution. The samples were then washed 3 times in standard saline. The acinus portion was extracted from the deep mammary gland tissue using eye scissors that had been disinfected in $75 \%$ alcohol, after which they were transferred into high-resistance PBS (containing $400 \mathrm{IU} / \mathrm{mL}$ of penicillin and $400 \mathrm{IU} / \mathrm{mL}$ of streptomycin). They were then placed in culture dishes on a clean bench, clarified using high-resistance and low-resistance PBS, disinfected for $30 \mathrm{~s}$ in $75 \%$ alcohol, and washed twice in $2 \times \mathrm{PBS}$ (PBS containing $200 \mathrm{IU} / \mathrm{mL}$ of penicillin and $200 \mathrm{IU} /$ $\mathrm{mL}$ of streptomycin). Following this, they were transferred to a $15-\mathrm{mL}$ tube, cut into 1 to $2 \mathrm{~mm}$ pieces, and finally cleaned and rinsed in the culture solution. The tissue pieces were tiled on the bottom of culture dish using a pincet and spearhead, inverted, and cultured in an incubator for $4 \mathrm{~h}$, inverted again, and cultured in the upright position while immersed in $1 \mathrm{~mL}$ of culture solution. Following this, $2 \mathrm{~mL}$ of culture solution was added and the sample was allowed to culture overnight. The solution was changed every 2 or $3 \mathrm{~d}$ to facilitate nutrient supply. Fibroblast cells dissociated from the tissue after $7 \mathrm{~d}$, and epithelial cells started to grow after $12 \mathrm{~d}$. When the cells became confluent, the fibroblasts and epithelial cells were isolated by difference using a trypsin digestion combined with a cell adherence speed method. Specifically, the cells were washed 3 times with PBS, followed by the addition of $0.25 \%$ trypsin and 3 min of digestion at $37^{\circ} \mathrm{C}$. The digestion was terminated by the addition of a solution containing serum. The round and levitating cells were discarded and the digestion was continued with trypsin. The cells were cultured for 2 to $3 \mathrm{~h}$, after which the cell solution was collected and the nonadherent cells were transferred to another culture dish.

This purification procedure was performed 2 to 3 times until the proportion of epithelial cells reached above $90 \%$, and these cells were used for subculture when $80 \%$ of these cells kept confluent. First, the entire primary culture solution was removed using a pipette. The epithelial cells were then washed 3 times with a small volume of $37^{\circ} \mathrm{C}$ PBS solution to remove any residual fetal bovine serum. Next, sufficiently warm $0.25 \%$ trypsin EDTA solution was added to the culture and then transferred to a plate placed on a warming tray heated to $37^{\circ} \mathrm{C}$ for $8 \mathrm{~min}$. The culture was observed using an inverted microscope to estimate whether the cells exhibited cell rounding, which is indicative of their detachment from the surface. Following this, the medium was removed from the dish and the suspension was pipetted into a $15-\mathrm{mL}$ conical tube containing 2 $\mathrm{mL}$ of complete medium. The cells were spun down and resuspended in further medium; $2 \times 10^{5} / \mathrm{mL}$ cell suspensions were then added to each of the fresh plates (appropriately labeled), and $2 \mathrm{~mL}$ of fresh medium was added to each new culture. Finally, the plates were in- 
cubated at $37^{\circ} \mathrm{C}$ in a $5 \% \mathrm{CO}_{2}$ incubator. The BMEC cells at generation 3 to 4 in the subculture were used for the infection and transfection studies.

\section{Infection and Transfection}

All cells were transfected by Lipofectamine 2000 (Invitrogen) according to the manufacturer's instructions (https://tools.thermofisher.com/content/sfs/manuals/ Lipofectamine_2000_Reag_protocol.pdf). First, 1 d before transfection, $2 \times 10^{5}$ cells were plated in $2 \mathrm{~mL}$ of growth medium lacking antibiotics to achieve 90 to $95 \%$ confluence at the time of transfection. Second, for each transfection sample, the complexes were prepared as follows: (a) dilute $5 \mu \mathrm{L}(50 \mu M), 7.5 \mu \mathrm{L}(75 \mu M)$, and $10 \mu \mathrm{L}$ of mimics $(100 \mu \mathrm{M}), 10 \mu \mathrm{L}$ of mimics NC $(100 \mu M)$, or $20 \mu \mathrm{L}$ of inhibitor $(20 \mu M)$ and $20 \mu \mathrm{L}$ of inhibitor $\mathrm{NC}(20 \mu M)$ in $250 \mu \mathrm{L}$ of OptiPro SFM and mix gently. (b) Mix Lipofectamine 2000 CD gently before use, and then dilute $5 \mu \mathrm{L}$ in $250 \mu \mathrm{L}$ of OptiPro SFM (Thermo Fisher Scientific) and incubate for 5 min at room temperature (note: diluted Lipofectamine 2000 CD must be combined with the diluted mimics or inhibitor within $30 \mathrm{~min}$ ). (c) After 5 min of incubation, combine the diluted mimics or inhibitor with diluted Lipofectamine $2000 \mathrm{CD}$ (total volume $=500 \mu \mathrm{L}$ ) and mix gently, incubating for $20 \mathrm{~min}$ at room temperature. Next, add $500 \mu \mathrm{L}$ of the complexes to each $60-\mathrm{mm}$ culture dish containing cells and $1.5 \mathrm{~mL}$ of medium lacking antibiotics and serum. Mix gently by rocking the plate back and forth. The medium was replaced by standard medium containing both antibiotics and serum after 4 to $6 \mathrm{~h}$. Finally, incubate the cells at $37^{\circ} \mathrm{C}$ in a $\mathrm{CO}_{2}$ incubator for 18 to $48 \mathrm{~h}$ before testing for transgene expression.

\section{Statistical Analysis}

All qRT-PCR results were calculated using a $2^{-\Delta \Delta C t}$ method (Livak and Schmittgen, 2001). Three independent technical replicates or biological replicates were processed for each test and each run was replicated in triplicate on a plate. Statistical significance was tested using SPSS 19.0 software (IBM Corp., Armonk, NY).

\section{RESULTS AND DISCUSSION}

\section{Construction of 2 Small RNA Libraries by Solexa Sequencing}

We created an extensive profile of miR expression in lactating and nonlactating buffalo mammary glands to identify miR that may play regulatory roles in lactation physiology, or may additionally have specific functional implications for fat synthesis. We were not able to harvest biopsy tissue to obtain 3 biological replicates for sequencing, as the mammary gland is prone to serious mastitis, resulting in a severe loss in milk production ability. Nevertheless, we attempted to biopsy the same buffalo at the 2 different stages of lactation, without success. We were not able to convince any other farmers to allow us to harvest biopsy tissue. Furthermore, the cost implication of slaughtering an additional buffalo was too high. Although we compared the sequencing data using only 1 lactating and 1 nonlactating buffalo, we were still able to obtain accurate information regarding differences in RNA expression between the lactating and nonlactating state as the RNA levels contrasted greatly between the 2 periods.

Solexa sequencing provided a total of $13,667,160$ reads from the library of the nonlactating tissues and 13,150,323 from the lactating mammary tissues. Low-quality reads, adaptors, and insufficiently tagged sequences were removed, resulting in a total of 12,569 , 467 , and $12,768,110$ reads of 18 to $31 \mathrm{nt}$, respectively. The total numbers of the small RNA sequences and high-quality reads in the 2 libraries are indicated in Table 1.

All of the Solexa sequencing reads were aligned against the Bos taurus genome (Btau_4.0) using the SOAP 3.0 Program (Li et al., 2008). Results matched $8,456,705$ reads and $7,665,749$ reads to the bovine genome in the nonlactating mammary library $(12,569,467$ reads in total) and in the lactating mammary library $(12,768,110$ reads in total; Table 1$)$, respectively. The buffalo small RNA in the 2 libraries were annotated to different categories (Table 1). Among these genomematched reads, 275,964 distinct reads were obtained for $\mathrm{miR}$ in the nonlactating library, whereas 254,130

Table 1. Expression profile of sequenced reads in 2 libraries constructed from lactating and nonlactating mammary glands of Bubalis bubalis

\begin{tabular}{llccr}
\hline Item & Reads & Distinct reads & Percentage & High-quality reads \\
\hline Lactating & Total & 922,005 & 100 & $12,768,110$ \\
& Perfect match to genome & 254,130 & 27.56 & $7,665,749$ \\
Nonlactating & Total & 840,949 & 100 & 60.04 \\
& Perfect match to genome & 275,964 & 32.82 & $12,569,467$ \\
$8,456,705$ & 67.28 \\
\hline
\end{tabular}

${ }^{1}$ Aligned with Bos taurus genome using soap2.20 (Li et al., 2008) aligner: SOAP. huge $-\mathrm{M} 0-\mathrm{v} 0-\mathrm{r} 2$. 


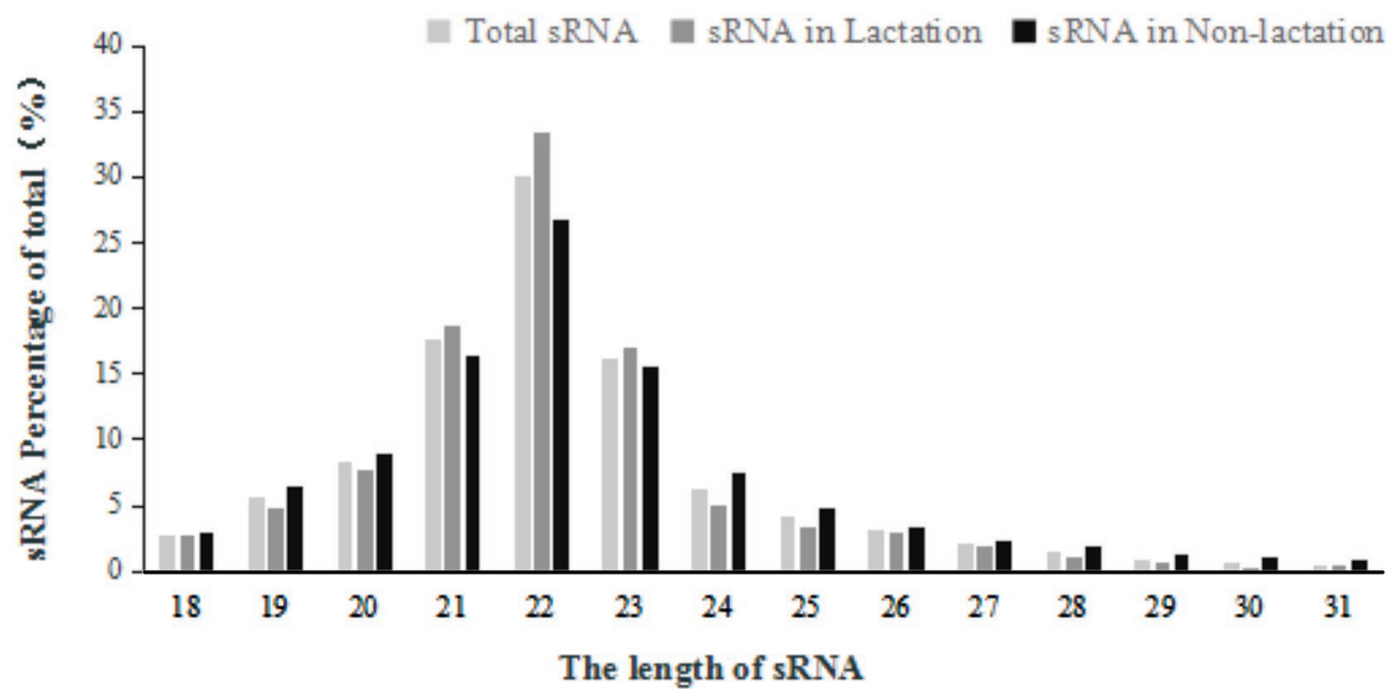

Figure 1. Length and frequency distribution of sequences (\% of total) in small RNA (sRNA) in the lactation and small RNA libraries of the buffalo mammary gland.

distinct reads were obtained for miR in the lactating library.

Of the total small sequences in the 2 libraries, the lengths of total small RNA increased in a graduated manner from 18 to $22 \mathrm{nt}$ where it peaked, which is typical of the small RNA of a Dicer-processed product (Figure 1). The size distribution of the small RNA from both tissues was 18 to $31 \mathrm{nt}$, with the 22 -nt sequences being the most common and accounting for $33.4 \%$ of the total (Figure 1).

The number of high-quality sequencing reads was less than the reads isolated from bovine mammary glands. However, as the bovine miR libraries were created for lactating and nonlactating mammary glands, there were more miR reads from lactating tissues than from nonlactating tissues, indicating that miR play an important role in lactation physiology ( $\mathrm{Li}$ et al., 2012b). In buffalo, about $33.4 \%$ of the RNA were $22 \mathrm{nt}$ in length, as compared with $52.6 \%$ in cows.

\section{Identification of Novel miR}

As the buffalo genome has not been completely sequenced, the bovine genome was searched for hairpin structures containing the candidate miR to determine whether these small RNA sequences are genuine buffalo miR. The MIREAP software was used to identify both known and novel miR from small RNA libraries deeply sequenced by Solexa technology. A total of 359 and 363 loci were confirmed as the known mature and pre-miR hairpins (676 mature miR and 662 pre-miR; miRbase V. 17.0) and matched in both the buffalo mammary libraries (Supplemental Table S5, https:// doi.org/10.3168/jds.2016-11461). A total of 259 families of known miR were expressed in both nonlactating and lactating mammary tissues (Table 2). After the removal of loci that overlapped with protein-coding gene exons and loci with free energy below $-18 \mathrm{kcal} /$ mol, the remaining 262 loci that had novel hairpins were considered candidate miR genes. More importantly, the identification of anti-sense miR from 35 novel miR candidates in the 2 tissues provided further evidence that they were novel miR. Of these 230 novel $\mathrm{miR}$ found in the buffalo mammary gland, 5 were here considered to be buffalo-specific because no homologs were found using miRBase V. 17.0. In general, most of the miR identified here are homologous to the known $\mathrm{miR}$ in this species, even with respect to close relatives, which supports the view that $\mathrm{miR}$ are evolutionarily conserved (Bartel, 2004).

Table 2. Detection statistics of conserved microRNA from lactating and nonlactating mammary gland tissue of Bubalus bubalis

\begin{tabular}{lc}
\hline Class & Bbu (Bubalus bubalis) \\
\hline Total mature microRNA & 676 \\
Confirmed by sequencing & 359 \\
No. of families ${ }^{1}$ & 259 \\
Total pre-microRNA & 662 \\
Confirmed by sequencing $^{1}$ No. of families & 363 \\
\hline
\end{tabular}

${ }^{1}$ There are 500 bta (Bos taurus) microRNA families in mirBase, version 17.0 (www.mirbase.org/). 
Analyses of the first nucleotide bias of the 17 to 29 nt miR candidates showed uridine (U) to be the most common (94.15 and 97.90\%) at the $5^{\prime}$ end of the 19- and 25-nt novel miR, whereas adenine (A) was the most common first nucleotide of the novel 20- (62.51\%), 23- (96.71\%), and 24-nt (50.72\%) miR in lactating mammary tissue (Supplemental Table S5, https://doi. org/10.3168/jds.2016-11461). In nonlactating mammary tissue, $\mathrm{U}$ was the dominant first nucleotide of the novel 19- (94.80\%), 25- (93.35\%), and 28-nt (100\%) miR, whereas A was the dominant first nucleotide of the $23-(94.59 \%)$ and 24 -nt $(97.62 \%)$ miR (Supplemental Table S6, https://doi.org/10.3168/jds.2016-11461).

The chromosome locations (Btau 4.0 as the most approximate template) for the known miR were investigated and searched for conserved and novel pre-miR via BLAST analysis in buffalo. Most of the novel miR were found on the buffalo autosomes. The genomic densities of the pre-miR were also analyzed (Supplemental Table S7, https://doi.org/10.3168/jds.2016-11461). Approximately $68.77 \%(240 / 349)$ of the known premiR that were successfully located in chromosomes in the mammary gland tissues were also found in the intergenic regions (Supplemental Table S8, https:// doi.org/10.3168/jds.2016-11461). According to Supplemental Table S8, the average density distribution of the known miR located in the autochromosome and $\mathrm{X}$ chromosome ranged from 0.09 to $1.05 \mathrm{miR}$ per $1 \mathrm{Mbp}$. the shortest chromosome, chr. 25, and longest chromosome, chr. 1, encoded 24 and $18 \mathrm{miR}$, respectively, corresponding to 0.54 and $0.11 \mathrm{miR}$ per $1 \mathrm{Mbp}$ genomic sequence. Chromosome 21 had the greatest number of known miR distributed (1.05 of pre-miR/Mb) in the buffalo genome as well as the expressed miR in the mammary gland (Supplemental Table S7, https://doi. org/10.3168/jds.2016-11461). The densities of the total miRNA distributed in autosome and $\mathrm{X}$ autosome were from 0.09 to $1.05 \mathrm{miR} / \mathrm{Mbp}$. Chromosome 21 was the maximum distribution chromosome, in which the density of total miR, miR in lactation, and miR in nonlactation were $1.05,1.34$, and $1.35 / \mathrm{Mbp}$ respectively. The densities of miR in the same chromosome between lactation and nonlactation were almost identical.

\section{Differential Expression of miR in Lactating and Nonlactating Mammary Tissue}

Solexa sequencing provided an important means of estimating the expression profiles of the miR genes by measuring the sequencing frequencies. The sequencing reads of the miR in the 2 mammary libraries are presented in Figure 2. The hierarchical clustering based on the relative cloning frequencies of the miR indicates that miR expression is different when the buffalo is lactating (Supplemental Figure S1 and Supplemental Table S5, https://doi.org/10.3168/jds.2016-11461).

In nonlactating tissues, bbu-miR-148a, bbu-let-7b, bbu-let-7a, bbu-miR-21, bbu-miR-143, bbu-miR-200c, bbu-miR-26a, bbu-miR-200a, and bbu-let-7f were the dominant expressed miR, with more than 20,000 reads. They constituted $53.8 \%$ of the total known miR sequencing reads, suggesting that they have abundant expression in nonlactating mammary tissue. The sequencing frequencies of the $57 \mathrm{miR}$ (e.g., bbu-miR92b, bbu-miR-421, bbu-miR-129, and so on) were much lower $(1 \leq$ sequencing reads $\leq 10)$. However, in lactating tissue, $8 \mathrm{miR}$ (bbu-let-7b, bbu-let-7a, bbumiR-26a, bbu-miR-125b, bbu-miR-21, bbu-miR-29a, and bbu-let-7c), each of which had more than 20,000 reads, were the most abundant. One hundred twentyfour miR (e.g., bbu-miR-34c, bbu-miR-2284c, and bbumiR-383) displayed the lowest sequencing frequencies (fewer than 10 reads). A comparison of the 2 libraries revealed that the expression of these miR, such as bbumiR-148a, bbu-miR-143, bbu-miR-200a, bbu-miR-141, and bbu-miR-30a-5p, decreased to less than half their nonlactating expression levels. Some miR, such as bbumiR-26a, bbu-miR-29a, bbu-miR-125b, bbu-let-7c, and bbu-miR-99a, displayed no less than twice the sequencing frequencies as the nonlactating mammary library. These results indicate that those miR whose expression changes following buffalo lactation might play a significant role in this process.

In the 2 mammary tissues, a total of 316 conserved miR were expressed in both types of mammary gland. Among the conserved miR, 9 miR, including bbu-miR-658, bbu-miR-2299-5p, bbu-miR-2448, bbumiR-2474, bbu-miR-329a, bbu-miR-202, bbu-miR-2367, bbu-miR-2484, and bbu-miR-208b, were specific to nonlactating tissue alone. Twenty miR, including bbu-miR2284k, bbu-miR-2325a, bbu-miR-2432, bbu-miR-2477, bbu-miR-449b, bbu-miR-1251, bbu-miR-2370*, bbumiR-1814c, bbu-miR-2310, bbu-miR-216b, bbu-miR219-3p, bbu-miR-2307, bbu-miR-2311, bbu-miR-2318, bbu-miR-2355*, bbu-miR-2370, bbu-miR-2378, bbumiR-2438, bbu-miR-2481, and bbu-miR-302a, were specific to lactating tissues alone. The Solexa sequencing results indicated that the different family members were expressed at markedly different levels. For example, the abundance of the bta-let- 7 family varied from 244 reads (bbu-let-7a*) to 806,206 reads (bbulet-7b) in the deep sequencing results in the lactating mammary tissues (Supplemental Table S9, https://doi. org/10.3168/jds.2016-11461). The expression levels of novel miR in the 2 libraries are shown in Supplemental Table S10 (https://doi.org/10.3168/jds.2016-11461). 


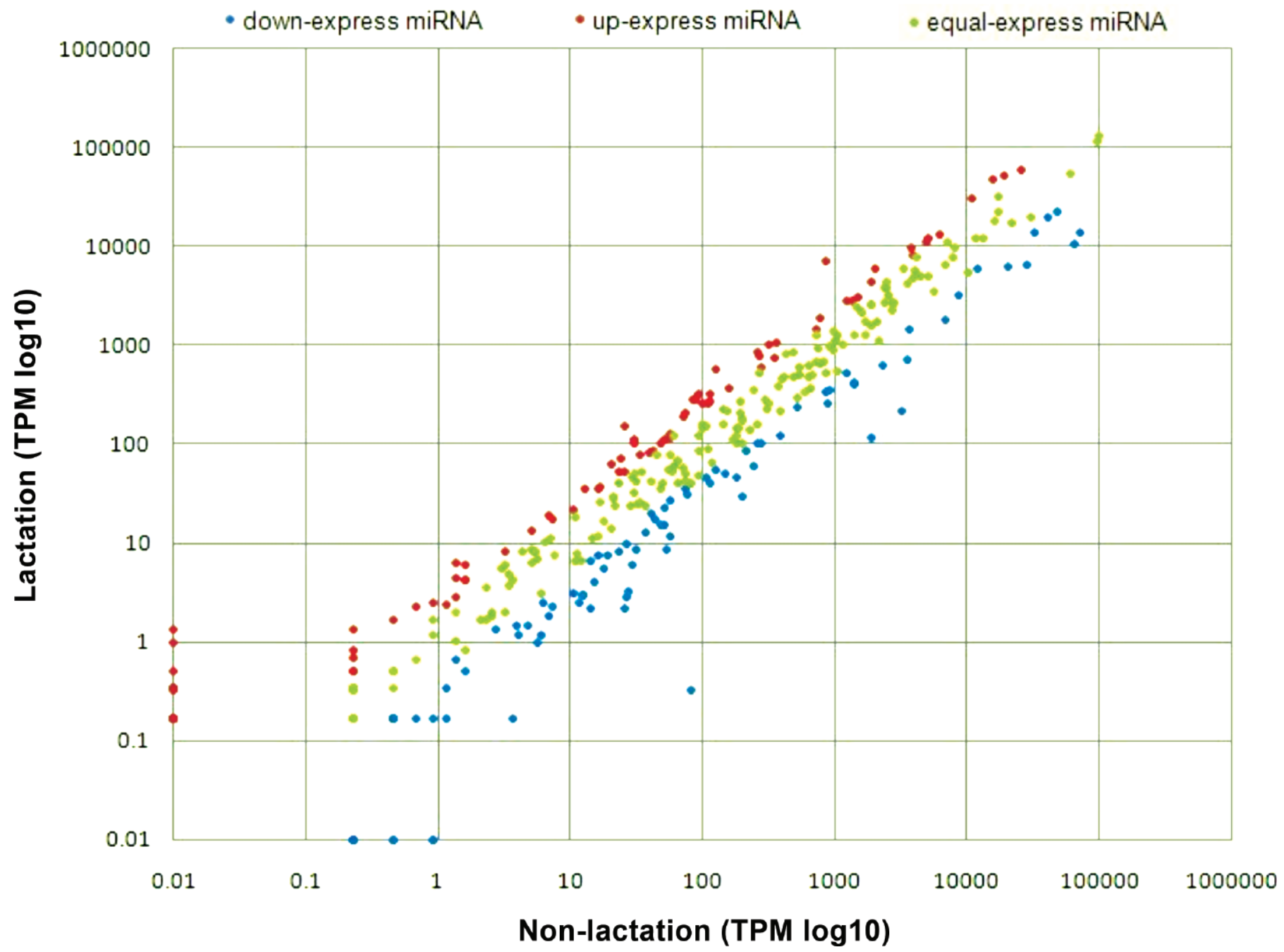

Figure 2. Comparison of levels of expression of microRNA (miRNA) in nonlactating (x-axis) and lactating (y-axis) mammary gland tissues. Red symbols represent miRNA with ratio $>2$; green symbols represent miRNA with ratio $>1 / 2$, but $<2$; blue symbols represent miRNA with ratio $<1 / 2$. Ratio $=$ normalized expression of the treatment/normalized expression of the control. TPM $=$ transcripts per million.

\section{Target Prediction and Signal Pathways Analysis}

To identify the potential target genes of the conserved and novel miR, target prediction was performed using MIREAP software. A total of 246,786 putative target sites for all the conserved miR, and 209,655 target sites for all the novel miR in the mammary tissue were identified (data not shown). The predicted target genes of the 20 differentially expressed genes with highly abundant miR were classified according to the Kyoto Encyclopedia of Genes and Genomes (KEGG) using functional annotations to identify pathways that were actively regulated by the conserved miR in mammary tissue (Supplemental Table S11, https://doi. org/10.3168/jds.2016-11461). Interestingly, most of the $109 \mathrm{miR}$ targets were part of the mitogen-activated pro- tein kinase signaling pathway, which plays an important role in almost every step of mammogenesis. The Janus kinase-signal transducer and activator of transcription signaling pathway was also found to be important and is known to be closely involved in mammalian lactation, especially in prolactin signal transduction (Watson and Burdon, 1996). One more significant pathway targeted by the highly expressed miR was the insulin signaling pathway, which is known to be involved in lactose metabolism and milk performance (Freeman et al., 2000).

\section{Validation of miR Expression with qRT-PCR}

The same RNA preparations used for the Solexa sequencing were subjected to qRT-PCR assay to validate 
the authenticity of the known and newly identified buffalo miR. The expression levels of $18 \mathrm{miR}$ were selected to be determined in both of the tissues.

Comparative analysis of the different miR expression profiles in the 2 tissues revealed 3 categories of miR: (1) 9 highly expressed conserved miR (bbu-miR-30a-5p, bbu-miR-141, bbu-miR-103, bbu-miR-101, bbu-miR30a-5p, bbu-miR-148a, bbu-miR-29a, bbu-miR-125b, bbu-miR-497, and bbu-miR-125a); (2) 4 miR with lowlevel expression in lactating and nonlactating tissues (bbu-miR-490, bbu-miR-217, bbu-miR-592, and bbumiR-2370*); and (3) 5 novel miR with high expression levels (novel-miR-57, novel-39, novel-148, novel-76, and novel-123b). The results of the qRT-PCR verification corresponded to the Solexa results. The expression of bbu-miR-103, bbu-miR-125a, bbu-miR-30a-5p, and bbu-miR-148a were 5.29-, 4.06-, 3.43-, and 2.43-fold higher $(P<0.05)$ in lactating than nonlactating tissues, respectively. Interestingly, the expression of bbu-miR-29a was found to be significantly lower $(P<$ 0.05 ), exhibiting a 0.3 -fold change in lactating versus nonlactating tissues. However, we found no significant difference $(P>0.05)$ in the expression levels of bbu-miR-141, bbu-miR-125b, bbu-miR-497, and bbumiR-101 recorded between these tissues (Figure 3A).

Among the miR with low-level expression, miR-490 and miR-592 were 4.96- and 3.82-fold higher in lactating than nonlactating tissues, respectively $(P<0.05$; Figure 3B). This was paralleled with the read values of 8:0 and 3:0 reads as assessed by Solexa sequencing in lactating and nonlactating tissue, and is therefore inconsistent with the results of the sequencing technology used. Among the 5 novel miR, we observed no significant differences $(P>0.05)$ between the novel-39, novel-148, novel-76, and novel-123b in lactating and nonlactating tissues. Interestingly, the expression of novel-miR-57 was 29.79-fold higher $(P<0.01)$ in lactating tissue than in nonlactating tissue (Figure $3 \mathrm{C}$ ). Based on these results, both miR-103 and novel-miR-57 were selected for further analysis of lactating and nonlactating tissues.

\section{Analysis of the Functional Role of Bubu-miR-103 in Milk Fat Synthesis}

Based on the vector backbone of the Lenti-Pac FIV Expression Packaging System (http://www.genecopoeia.com/), the vector containing the precursor of the bta-mir-103-1 clone was constructed and named LpEZX-pre-MR103 (HIV; Supplemental Figure S2, https://doi.org/10.3168/jds.2016-11461). The plasmid was 8,219 bp in size; it was packaged in $293 \mathrm{~T}$ cell lines (Supplemental Figure S3, https://doi.org/10.3168/ jds.2016-11461) and the infection titer was determined to be $3.42 \times 10^{6}$ and $3.47 \times 10^{6} \mathrm{PFU}$ (transcripts per million) $/ \mathrm{mL}$ of $\mathrm{NC}$ and miR-103, respectively, on 293T cell lines (Supplemental Figure S4, https://doi. org/10.3168/jds.2016-11461). The miRNA inhibitor was synthesized; BMEC were then cultured in tissue blocks and infected using a lentiviral vector with miR-103. The inhibitor of miR-103 was transfected at the same time (Supplemental Figure S5, https://doi. org/10.3168/jds.2016-11461). The overexpression and suppression of miR-103 were negatively correlated with pantothene kinase 3 (PANK3). Hence, the target of miR-103 was confirmed as PANK3 (Figure 4A). The overexpression of miR-103 combined with the $3^{\prime}$ untranslated region of $P A N K 3$ downregulated the expression of $P A N K 3$, upregulated the expression of acetyl CoA carboxylase $\alpha$ (ACACA; Figure 4B), and significantly enhanced the expression of the steroid regulatory element binding protein (SREBP1c; Figure 4C). The effects of overexpression of miR-103 on the 8 key fat synthesis metabolism pathways were determined. The results indicated that bbu-miR-103 enhances fatty acid synthesis through de novo synthesis and also plays a role in triglyceride synthesis, fat droplet formation and secretion, as well as fatty acid absorption. However, it does not have a significant effect on fatty acid transfer metabolism (Table 3).

The $3 \mathrm{miR}$ of the miR-103 family were transcribed from the fifth intron of PANK3. Water buffalo MiR103-1 was found to be targeted to PANK3 to enhance milk fat synthesis, which is in accordance with published reports on dairy goats (Lin et al., 2013); PANK3 is a key regulating enzyme in the biosynthesis of CoA (Rock et al., 2000). Acetyl CoA is involved in de novo fatty acid synthesis (Robishaw and Neely, 1985). The overexpression of miR-103 reduces the expression of $P A N K 3$, which in turn relieves the inhibitory effect on acetyl CoA and promotes acetyl CoA to provide more substrates for fatty acid synthesis, enhancing de novo fatty acid synthesis. The FASN and ACACA enzymes are the main pathways involved in de novo synthesis of fatty acids (Lin et al., 2013), and our study showed that miR-103 enhanced the FASN pathway indirectly. The major transcription factors that modulate the expression of several enzymes implicated in fatty acid and triacylglycerol synthesis are $S R E B P 1 c$, peroxisome proliferator-activated receptor- $\gamma$, and liver receptor $\mathrm{X}-\alpha$ (Desvergne et al., 2006). In our study, the overexpression of miR-103 significantly improved the expression of $S R E B P 1 c$, indicating that miR-103 not only affected the target gene pank3, but also indirectly upregulated the transcription factors leading to an increase in the expression of downstream genes. 
A

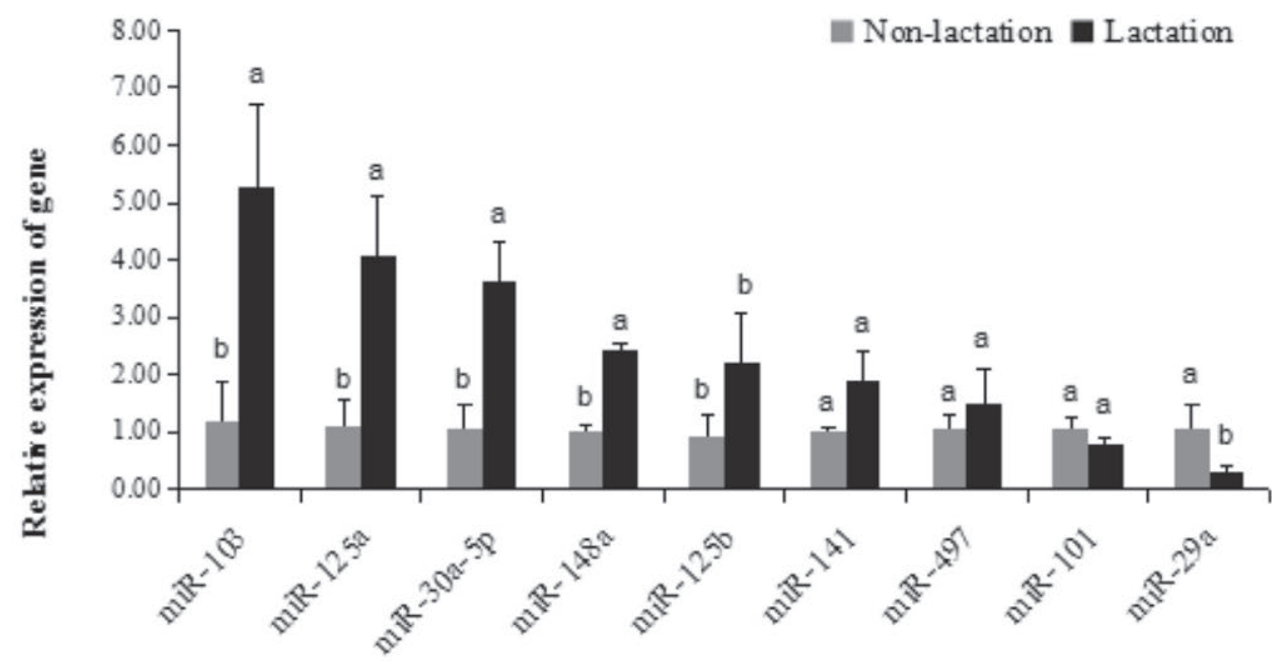

Conserved miR with high expression

B

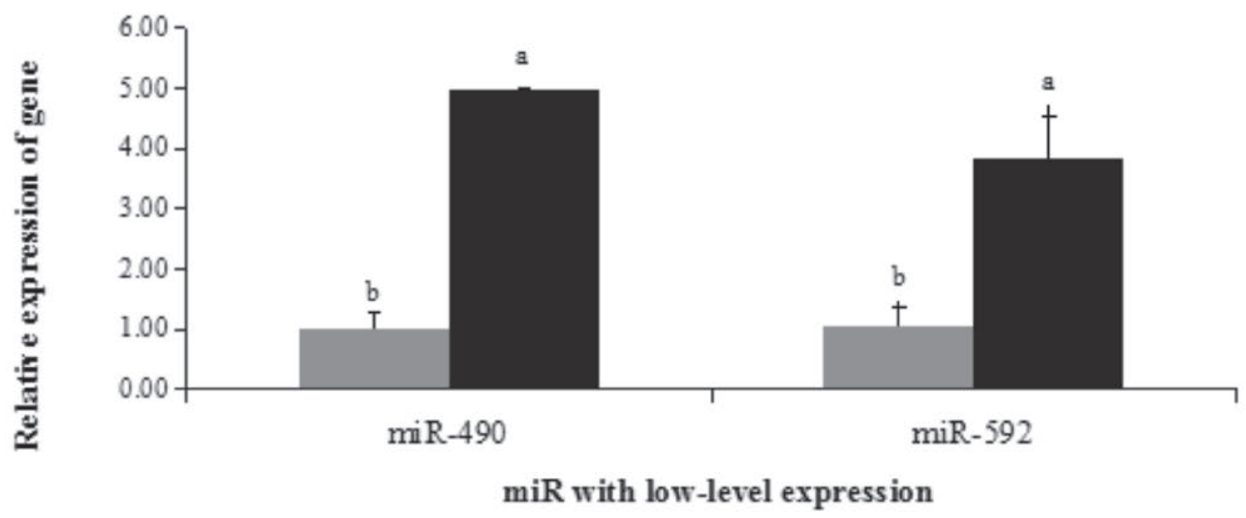

C

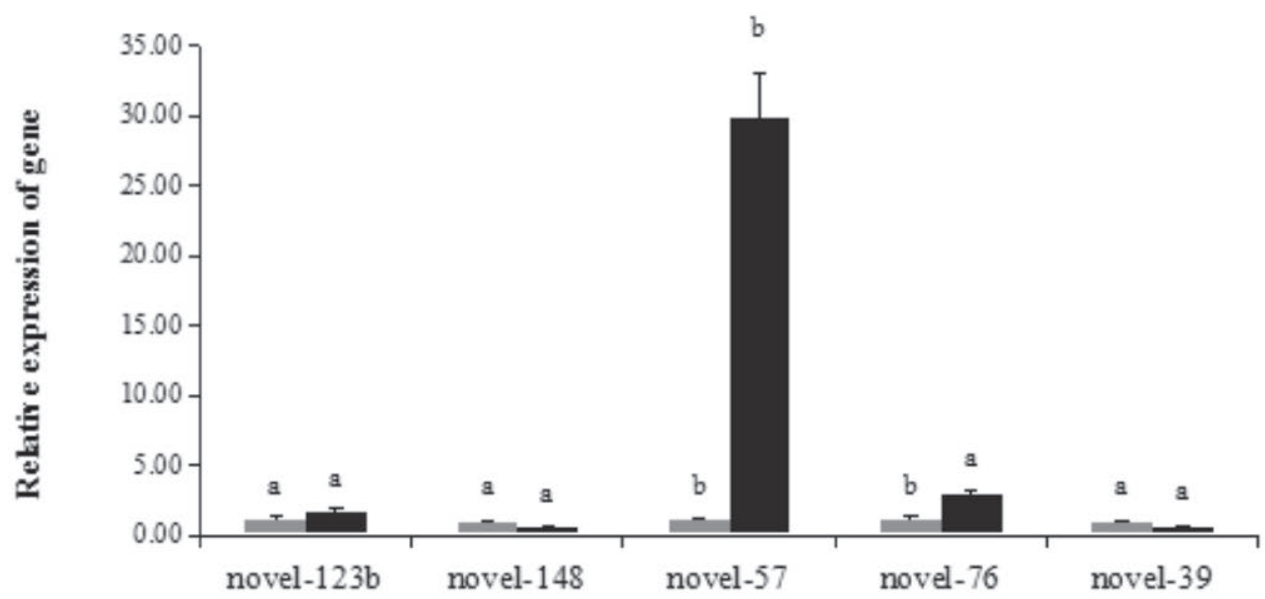

Novel miR with high-level expression

Figure 3. Quantitative real-time PCR results of (A) conserved highly abundant microRNA (miR), (B) low-abundance miR, and (C) novel$\mathrm{miR}$ in Bubalus bubalis lactating and nonlactating mammary glands. These data show the means \pm SD from 3 independent technical replicates for each buffalo. Different letters above bars indicate a significant difference between the groups $(P<0.05)$, and the same letter indicates no significant difference between the groups $(P>0.05)$. 
A

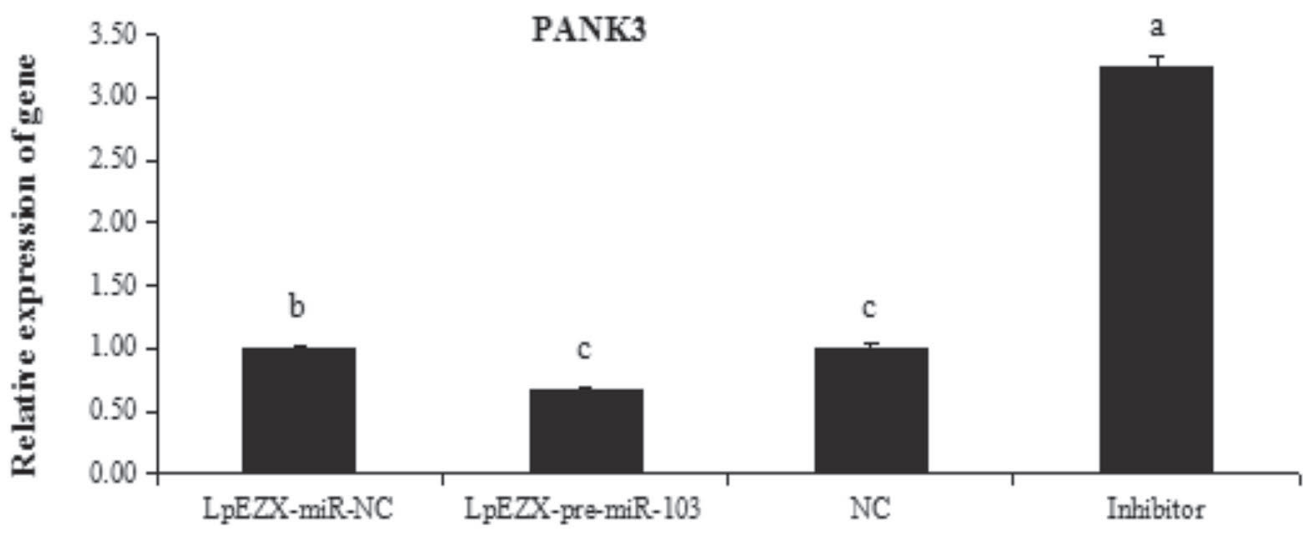

B

\section{ACACA}

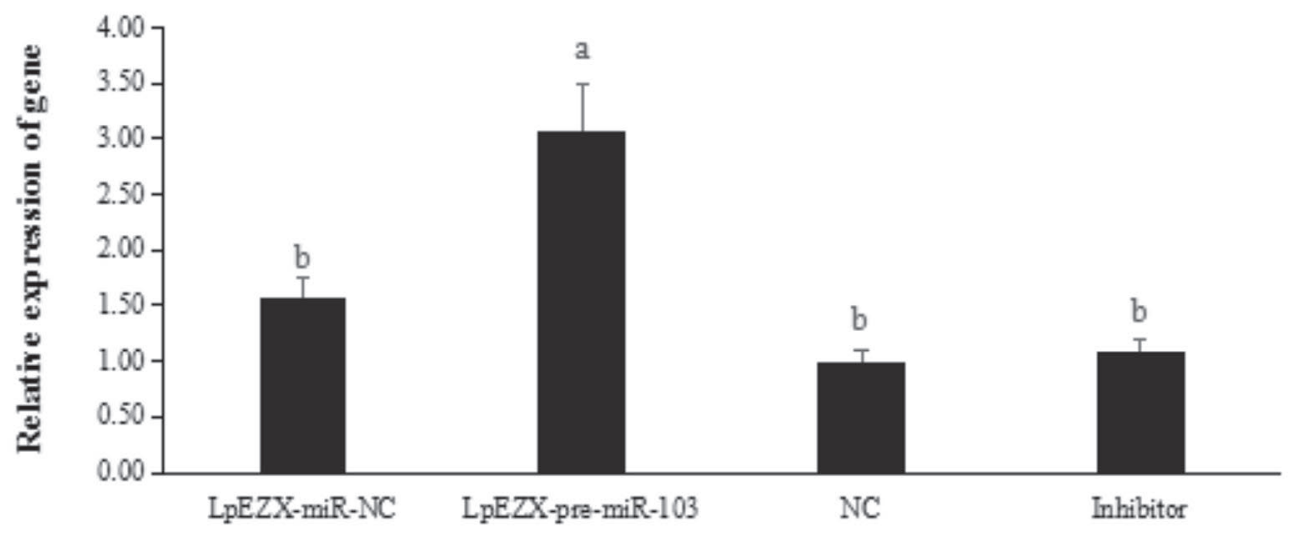

C

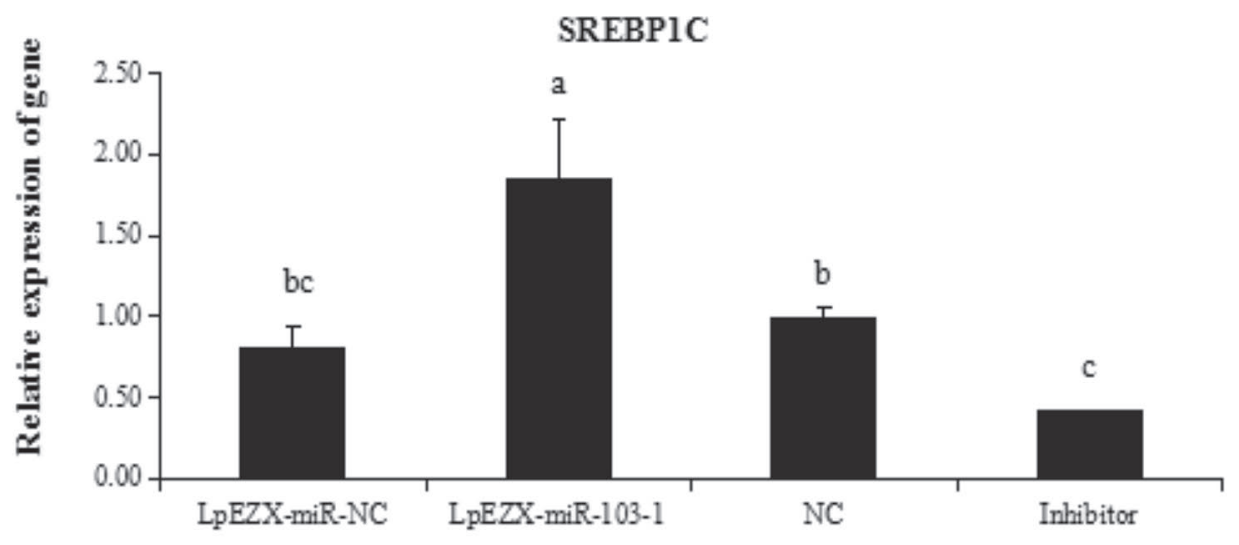

\section{Groups}

Figure 4. Quantitative real-time PCR indicating relative gene expression of (A) pantothene kinase 3 (PANK3), (B) acetyl CoA carboxylase $\alpha(A C A C A)$, and $(\mathrm{C})$ steroid regulatory element binding protein $(S R E B P 1 c), \beta$-actin served as the endogenous control for genes involved in milk fat pathway relating to bbu-miR-103. $\mathrm{NC}=$ negative control. PANK3 was downregulated and upregulated by LpEZX-pre-miR-103 and inhibitor comparing to LpEZX-miR-NC and NC, respectively. The relative expression of $A C A C A$ and $S R E B P 1 c$ was separately upregulated by LpEZX-pre-miR-103 and LpEZX-miR-NC. These data show the means \pm SD from 3 independent biological replicates for each cell line. Different letters above bars indicate a significant difference between the groups $(P<0.05)$, and the same letter indicates no significant difference between the groups $(P>0.05)$. 


\section{Analysis of Novel-miR-57 and the Role of DOK4}

Novel-miR-57 was the most significantly differentially expressed miR of the novel miR in both lactating and nonlactating tissues. The secondary structure of novel-miR-57 was predicted by MiRscan (http://genes. mit.edu/mirscan/), and the results showed that 7 stemloops existed with a binding free energy of $-28.0 \mathrm{kcal} /$ mol. The mature sequence of novel-miR- 57 was located on the first stem-loop. The $3^{\prime}$ untranslated regions of annotated buffalo mRNA were intercepted by the self-programming software Ensembl V. 80 (http://asia. ensembl.org/Bos_taurus/Info/WhatsNew?db=core; $\mathrm{g} \%$ 3DENSBTAG00000000941;r\%3D2:1850107-1899289;t\% 3DENSBTAT00000001246), as a database for predicting the target gene of bbu-miR-57.

The mRNA was considered to be a target gene if its binding free energy to the 3 ' untranslated region of the 2 to 8 seed sequence of the novel-miR-57 was below -20 $\mathrm{kcal} / \mathrm{mol}$. Finally, 34 buffalo mRNA were identified as the possible targets of novel-miR-57, including CYP7B1, CACNG3, DOK4, COL17A1, ESN1, and others. The Gene Ontology assignment and KEGG pathway analysis of the 34 target mRNA was predicted. The nematode miR-39 served as the external control and the significantly different target genes of novel-miR-57 were searched by consulting the literature, designing specific primers, as well as via qRT-PCR analysis in lactating and nonlactating mammary gland tissues. Six genes were not detected by qRT-PCR; the remaining genes were divided into 2 groups if the fold change in relative gene expression was reflected by a 2 - or 3 -digit number. Accordingly, the fold change in gene relative expression levels of 10 genes, including $R C L 1, U B E 3 C$, and $N F R K B$, in nonlactating tissues were 0.98 to 10.59 to that in lactating tissue (Figure $5 \mathrm{~A}$ ), whereas the expression levels of 11 genes, including $B R M S 1 L$, $A C T L, A D O R O, R C L 1, U B E 3 C$, and NFRKB, in lactating tissue were higher than or equal to that in nonlactating tissues (Figure 5B). The most significantly differentially expressed genes included DLX3 (128.03fold), CANCNG3 (144.23-fold), DOK4 (146.24-fold), NFKBID (160.96-fold), C17orf53 (160.38-fold), RTN1 (274.4-fold), and FBXO10 (326.24-fold). The relative expression levels of these genes were far higher in nonlactating tissues than in lactating tissue $(P<0.01$; Figure $5 \mathrm{C}$ ). The mimics and inhibitor of novel-miR-57 were chemically synthesized, after which 50,75 , and $100 \mathrm{n} M$ of novel-miR-57 mimics and $200 \mathrm{n} M$ of inhibitor were individually transfected into Bcap-37 cell lines, which

Table 3. Major milk fat synthesis pathways and individual genes affected by overexpression of microRNA-103 targeted to pantothene kinase 3 in buffalo mammary epithelial cell

\begin{tabular}{|c|c|}
\hline Gene symbol & miR-103-infected cells \\
\hline \multicolumn{2}{|l|}{ 1. De novo fatty acid synthesis } \\
\hline FASN (fatty acid synthase) & $0.44 \pm 0.28$ \\
\hline$A C A C A$ (acetyl-coenzyme $\mathrm{A}$ carboxylase $\alpha)$ & $3.06 \pm 0.43^{* *}$ \\
\hline ACSS2 (acetyl-coenzyme A synthetase, cytoplasmic) & $1.00 \pm 0.09$ \\
\hline$S R E B P 1 c$ (sterol regulatory element binding protein) & $1.86 \pm 0.35^{*}$ \\
\hline LXRa (liver X receptor $\alpha$ ) & $1.25 \pm 0.28$ \\
\hline \multicolumn{2}{|l|}{ 2. Triglyceride synthesis } \\
\hline$S C D$ (stearoyl-CoA desaturase) & $1.12 \pm 0.22^{*}$ \\
\hline GPAM (glycerol-3-phosphate acyltransferase, mitochondrial) & $3.76 \pm 0.48^{* *}$ \\
\hline AGPAT6 (1-acylglycerol-3-phosphate O-acyltransferase 6) & $1.05 \pm 0.45$ \\
\hline DGAT1 (diacylglycerol O-acyltransferase 1 ) & $2.33 \pm 0.02^{* *}$ \\
\hline LEPIN1 (lipid phosphate phosphohydrolase1) & $1.58 \pm 1.73$ \\
\hline \multicolumn{2}{|l|}{ 3. Milk fat droplet formation and secretion } \\
\hline$A D F P$ (adipocyte differentiation-related protein) & $1.61 \pm 0.36^{*}$ \\
\hline TIP 47 (tail-interacting protein 47 ) & $1.02 \pm 0.26$ \\
\hline gBTNIA1 (butyrophilin) & $1.14 \pm 0.78$ \\
\hline \multicolumn{2}{|l|}{ 4. Fatty acid uptake } \\
\hline CD36 (transmembrane protein CD36) & $1.96 \pm 0.36^{*}$ \\
\hline \multicolumn{2}{|l|}{ 5. Fatty acid activation } \\
\hline$A C L Y$ (ATP-citrate lyase) & $1.00 \pm 0.10$ \\
\hline ACSS1 (short-chain acyl CoA dehydrogenase) & $1.20 \pm 0.43^{*}$ \\
\hline PDK4 (pyruvate dehydrogenase kinase 4) & $4.74 \pm 0.45^{* *}$ \\
\hline \multicolumn{2}{|l|}{ 6. Lipolysis and release } \\
\hline CPT1 (carnitine palmitoyl transterase- 1 ) & $1.00 \pm 0.06$ \\
\hline$A T G L$ (adipose triglyceride lipase) & $1.01 \pm 0.20$ \\
\hline \multicolumn{2}{|l|}{ 7. Fatty acid oxidation } \\
\hline$A M P K \alpha$ (adenosine $5^{\prime}$-monophosphate-activated protein kinase) & $1.00 \pm 0.13$ \\
\hline \multicolumn{2}{|l|}{ 8. Fatty acid receptors } \\
\hline GPK41 (G protein-coupled receptors 41$)$ & - \\
\hline$D G A T$ (diacylglycerol acyltransferase) & $1.00 \pm 0.06$ \\
\hline
\end{tabular}


A

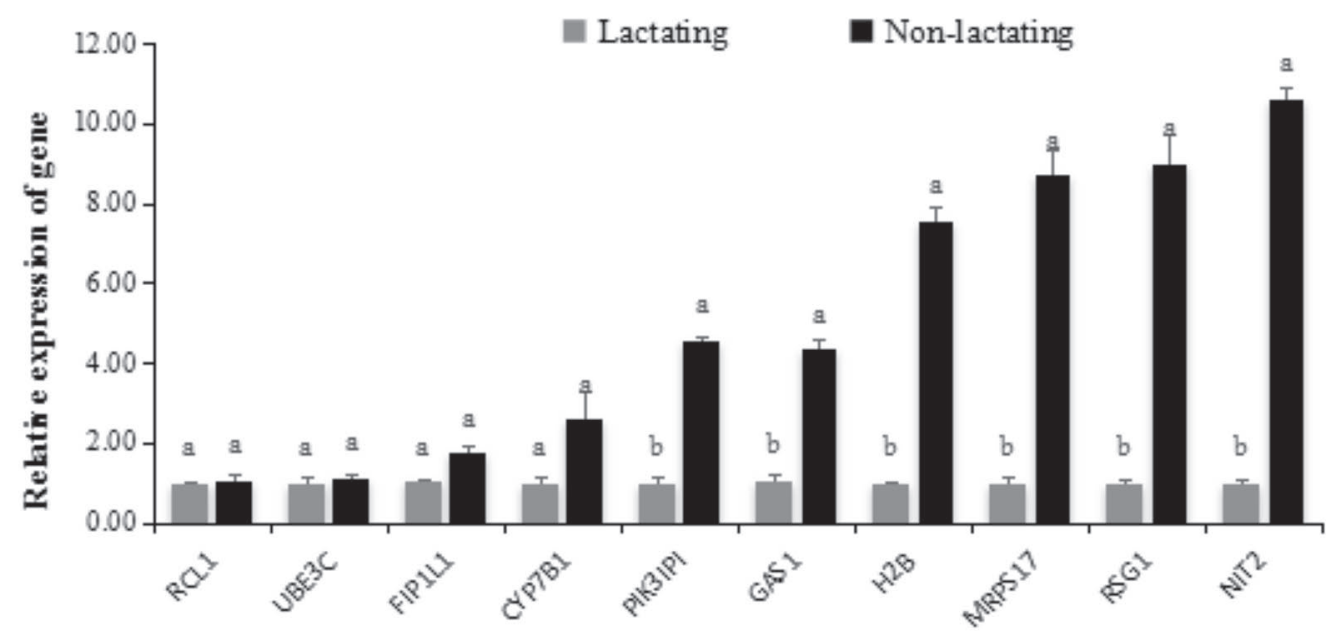

B

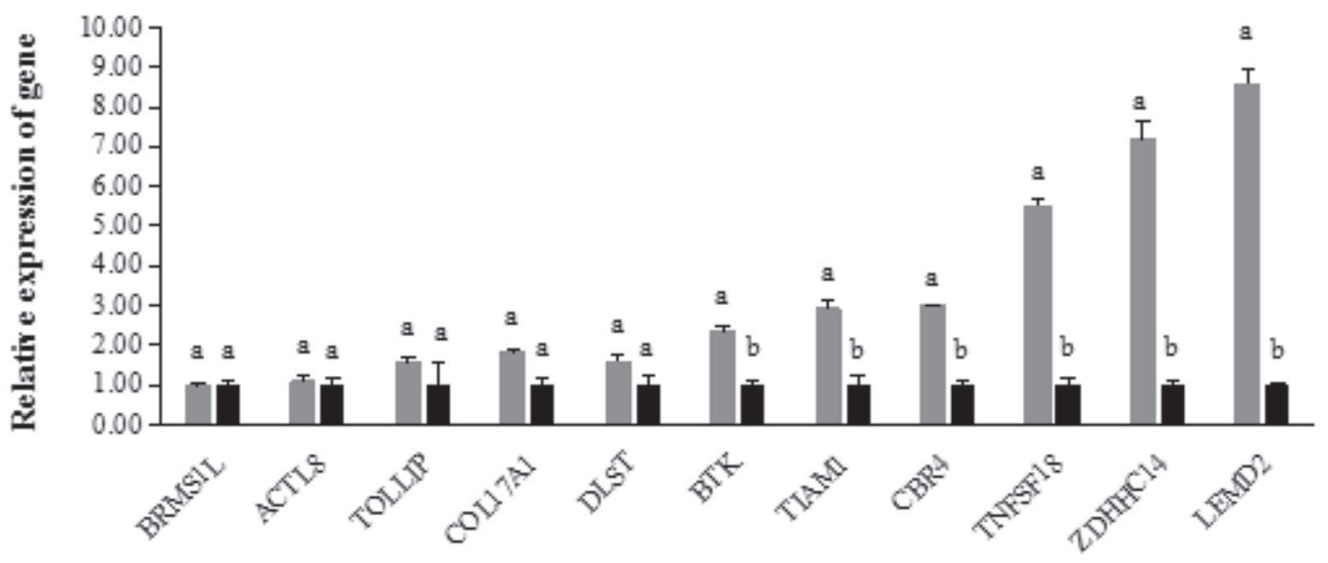

$\mathbf{C}$

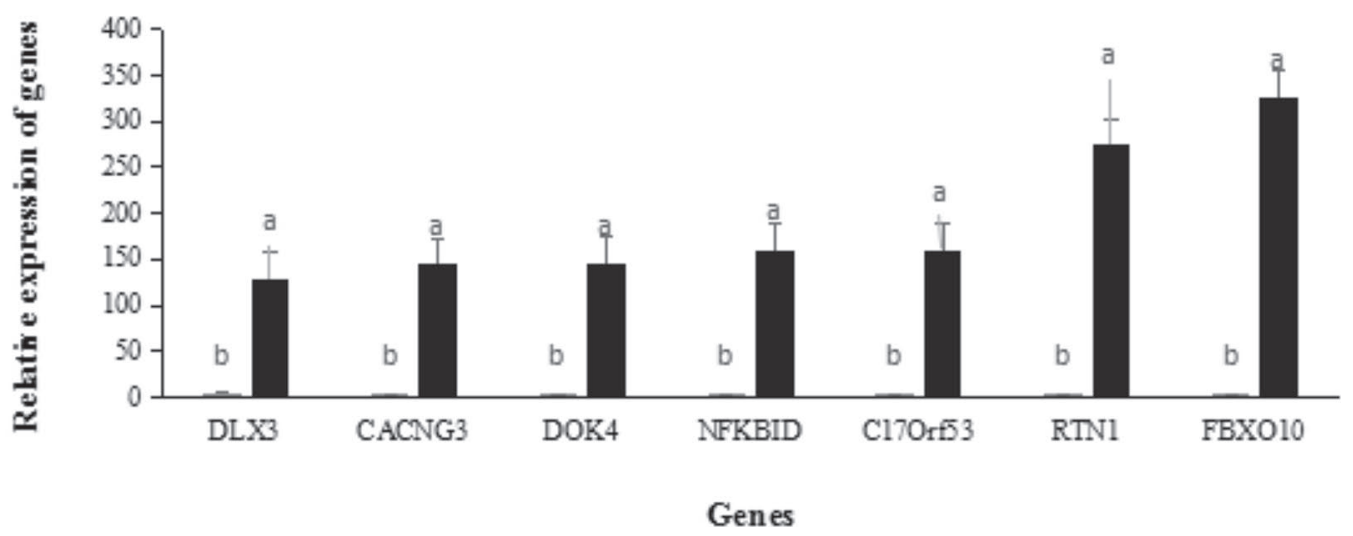

Figure 5. Quantitative real-time PCR analysis of differential gene expression in lactating and nonlactating mammary glands of Bubalus bubalis. (A) Genes in which expression in nonlactating mammary glands was higher than or equal to that in lactating mammary glands, (B) genes in which expression in nonlactating mammary glands was lower than or equal to that in lactating mammary glands, and (C) genes in which expression in nonlactating mammary glands was substantially higher than in lactating mammary glands. These data show the means \pm SD from 3 independent technical replicates for each buffalo. Different letters above bars indicate a significant difference between the groups $(P$ $<0.05)$, and the same letter indicates no significant difference between the groups $(P>0.05)$. 
A

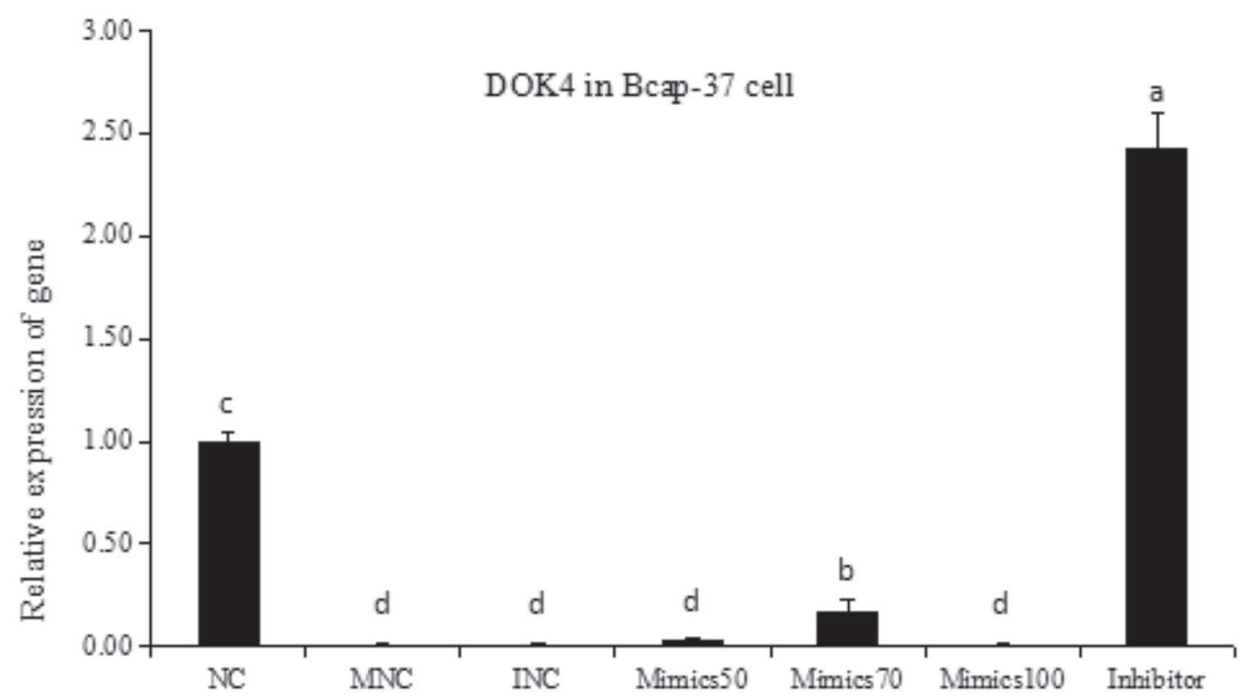

B

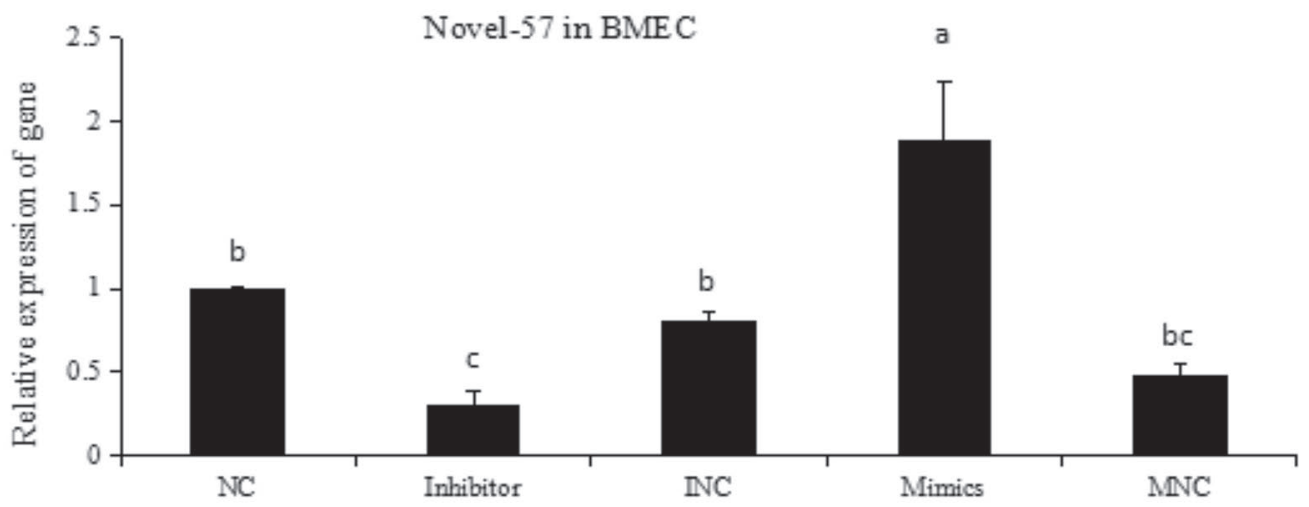

C

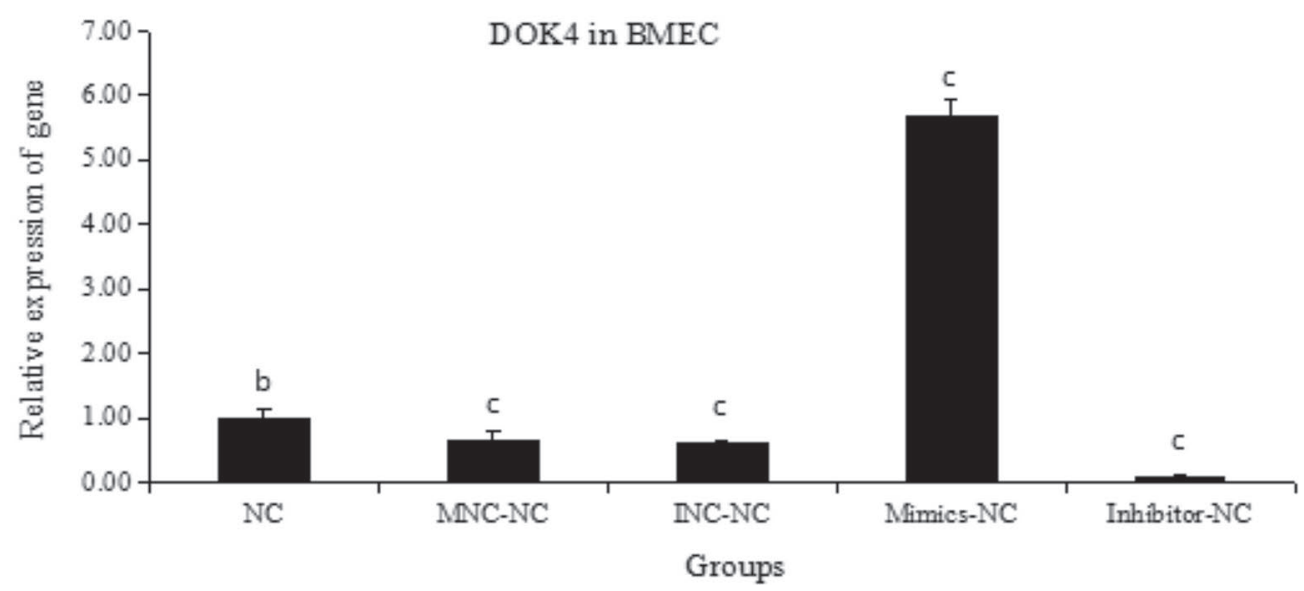

Figure 6. Functional analysis of novel-miR-57. (A) Effect on docking protein 4 (DOK4) expression in Bcap-37 cells in the presence of various concentrations $(50,70$, and $100 \mathrm{n} M$ ) of mimics or $200 \mathrm{n} M$ of inhibitor. $\mathrm{NC}=$ negative control. (B) Expression of novel-miR-57 in buffalo mammary epithelial cells (BMEC) after transfection in presence of mimics or inhibitor; (C) effects on DOK4 expression in BMEC following transfection in presence of mimics and inhibitor. These data show the means \pm SD from 3 independent biological replicates for each cell line. $\mathrm{MNC}=$ mammary NC; INC $=$ inhibitor NC. Different letters above bars indicate a significant difference between the groups $(P<0.05)$, and the same letter indicates no significant difference between the groups $(P>0.05)$. 
indicated that, of the 7 genes, only $D_{0 K} 4$ was related to novel-miR-57 (Figure 6A). The expression of DOK4 was extremely significantly increased or decreased by novel-miR-57 inhibitor and mimics, respectively $(P<$ 0.01; Figure 6B). When BMEC were transfected by the same $100 \mathrm{n} M$ mimics or $200 \mathrm{n} M$ of inhibitor of novelmiR-57, the gene expression of $D^{O K K} 4$ was significantly promoted or reduced, respectively $(P<0.01$; Figure $6 \mathrm{C})$. This was opposite of what was observed in the Bcap-37 cell line. We concluded that DOK4 was the putative target gene in both Bcap-37 cell line and BMEC, and that the novel-miR-57 could selectively upregulate or downregulate the expression of $\mathrm{DOK}_{4}$ according to different physiological conditions and ultimate function in the lactation metabolism pathway. This is not the only exception to the upregulation of gene function among miR (Vasudevan et al., 2007), nor is it the first time opposite effects of same miR in different cell lines have been discovered. For example, miR-24 was found to downregulate cell-growth in A549 cells, but increase cell growth in HeLa cells (Cheng et al., 2005). In epithelial cells, DOK4 is a promiscuous inhibitor of tyrosine kinase signaling induced by differentiation. High abundances of novel-miR-57 can enhance DOK4 expression and promote differentiation of BMEC, which could confer a positive effect on lactation physiology. The precise mode of $\mathrm{DOK}_{4}$ action in lactation physiology remains to be elucidated.

\section{ACKNOWLEDGMENTS}

This study was supported by a grant from the China National High Technology Research and Development Program (863) Project (2011AA100607), National Natural Science Foundation Project (31260552), and Guangxi Scientific Research and Technological Development project (GuiKeHe14125008-2-13).

\section{REFERENCES}

Altschul, S. F., W. Gish, W. Miller, E. W. Myers, and D. J. Lipman. 1990. Basic local alignment search tool (BLAST). J. Mol. Biol. 215:403-410.

Audic, S., and J. M. Claverie. 1997. The significance of digital gene expression profiles. Genome Res. 7:986-995.

Bartel, D. P. 2004. MicroRNAs: Genomics, biogenesis, mechanism, and function. Cell 116:281-297. https://doi.org/10.1016/S00928674(04)00045-5.

Brennecke, J., D. R. Hipfner, A. Stark, R. B. Russel, and S. M. Cohen. 2003. Bantam encodes a developmentally regulated microRNA that controls cell proliferation and regulates apoptotic gene hid in Drosophila. Cell 113:25-36. https://doi.org/10.1016/S00928674(03)00231-9.

Bu, D. P., X. M. Nan, F. Wang, J. J. Loor, and J. Q. Wang. 2015. Identification and characterization of microRNA sequences from bovine mammary epithelial cells. J. Dairy Sci. 98:1696-1705. https://doi.org/10.3168/jds.2014-8217.
Caraux, G., and S. Pinloche. 2005. PermutMatrix: A graphical environment to arrange gene expression profiles in optimal linear order. Bioinformatics 21:1280-1281. https://doi.org/10.1093/ bioinformatics/bti141.

Carrington, J. C., and V. Ambros. 2003. Role of microRNAs in plant and animal development. Science 301:336-338. https://doi. org/10.1126/science.1085242.

Cheng, A. M., M. W. Byrom, J. Shelton, and L. P. Ford. 2005. Antisense inhibition of human miRNAs and indications for an involvement of miRNA in cell growth and apoptosis. Nucleic Acids Res. 33:1290-1297. https://doi.org/10.1093/nar/gki200.

Desvergne, B., L. Michalik, and W. Wahli. 2006. Transcriptional regulation of metabolism. Physiol. Rev. 86:465-514. https://doi. org/10.1152/physrev.00025.2005.

Freeman, M. E., B. Kanyicska, A. Lerant, and G. Nagy. 2000. Prolactin: Structure, function, and regulation of secretion. Physiol. Rev. 80:1523-1631.

Glazov, E. A., P. A. Cottee, W. C. Barris, R. J. Moore, B. P. Dalrymple, and M. L. Tizard. 2008. A microRNA catalog of the developing chicken embryo identified by a deep sequencing approach. Genome Res. 18:957-964.

Griffiths-Jones, S., H. K. Saini, S. van Dongen, and A. J. Enright. 2008. miRBase: Tools for microRNA genomics. Nucleic Acids Res. $36: 154-158$.

Hafner, M., P. Landgraf, J. Ludwig, A. Rice, T. Ojo, C. Lin, D. Holoch, C. Lim, and T. Tuschi. 2008. Identification of microRNAs and other small regulatory RNAs using cDNA library sequencing. Methods 44:3-12.

Ji, Z., G. Wang, Z. Xie, J. Wang, C. Zhang, F. Dong, and C. Chen. 2012a. Identification of novel and differentially expressed micrornas of dairy goat mammary gland tissues using solexa sequencing and bioinformatics. PLoS One 7:e49463. https://doi.org/10.1371/ journal.pone.0049463.

Ji, Z., G. Z. Wang, Z. J. Xie, C. L. Zhang, and J. Wang. 2012b. Identification and characterization of microRNAs in the dairy goat (Capra hircus) mammary gland by Solexa deep-sequencing technology. Mol. Biol. Rep. 39:9361-9371. https://doi.org/10.1007/ s11033-012-1779-5.

Johnson, G., T. Nolan, and S. A. Bustin. 2013. Real-time quantitative PCR, pathogen detection and miqe. Methods Mol. Biol. 943:1-16.

Le Quesne, J., and C. Caldas. 2010. Micro-RNAs and breast cancer. Mol. Oncol. 4:230-241. https://doi.org/10.1016/j.molonc.2010.04.009.

Lewis, B. P., I. H. Shih, M. W. Jones-Rhoades, D. P. Bartel, and C. B. Burge. 2003. Prediction of mammalian microRNA targets. Cell 115:787-798. https://doi.org/10.1016/S0092-8674(03)01018-3.

Li, R., Y. Li, K. Kristiansen, and J. Wang. 2008. SOAP: Short oligonucleotide alignment program. Bioinformatics 24:713-714. https:// doi.org/10.1093/bioinformatics/btn025.

Li, Y., Z. Zhang, F. Liu, W. Vongsangnak, Q. Jing, and B. Shen. 2012a. Performance comparison and evaluation of software tools for microRNA deep-sequencing data analysis. Nucleic Acids Res. 40:4298-4305. https://doi.org/10.1093/nar/gks043.

Li, Z., X. Lan, W. Guo, J. Sun, Y. Huang, J. Wang, T. Huang, C. Lei, X. Fang, and H. Chen. 2012c. Comparative transcriptome profiling of dairy goat microRNAs from dry period and peak lactation mammary gland tissues. PLoS One 7:e52388. https://doi. org/10.1371/journal.pone.0052388.

Li, Z., H. Liu, X. Jin, L. Lo, and J. Liu. 2012b. Expression profiles of microRNAs from lactating and non-lactating bovine mammary glands and identification of miRNA related to lactation. BMC Genomics 13:731. https://doi.org/10.1186/1471-2164-13-731.

Lim, L. P., M. E. Glasner, Y. Soraya, C. B. Burge, and D. P. Bartel. 2003. Vertebrate microRNA genes. Science 299:1540. https://doi. org/10.1126/science.1080372.

Lin, X., J. Luo, L. Zhang, and J. Zhu. 2013. MicroRNAs synergistically regulate milk fat synthesis in mammary gland epithelial cells of diary goats. Gene Expr. 16:1-13. https://doi.org/10.3727/1052 21613X13776146743262.

Liu, C. G., G. A. Calin, B. Meloon, N. Gamliel, C. Sevignani, M. Ferracin, C. D. Dumitru, M. Shimizu, S. Zupo, M. Dono, H. Alder, F. Bullrich, M. Negrini, and C. M. Croce. 2004. An oligonucleotide 
microchip for genome-wide microRNA profiling in human and mouse tissues. Proc. Natl. Acad. Sci. USA 101:9740-9744. https:// doi.org/10.1073/pnas.0403293101.

Livak, K. J., and T. D. Schmittgen. 2001. Analysis of relative gene expression data using real time quantitative $\mathrm{PCR}$ and the $2^{-\Delta \Delta \mathrm{CT}}$ method. Methods 25:402-408. https://doi.org/10.1006/ meth.2001.1262.

Miao, L., H. Yao, C. Li, M. Pu, X. Yao, H. Yang, X. Qi, J. Ren and Y. Wang. 2016. A dual inhibition: MicroRNA-552 suppresses both transcription and translation of cytochrome P450 $2 \mathrm{E} 1$. Biochim. Biophys. Acta 1859:650-662. https://doi.org/10.1016/j. bbagrm.2016.02.016.

Robishaw, J. D., and J. R. Neely. 1985. Coenzyme A metabolism. Am. J. Physiol. 248:E1-E9. http://www.ncbi.nlm.nih.gov/ pubmed $/ 2981478$

Rock, C. O., R. B. Calder, M. A. Karim, and S. Jackowski. 2000 Pantothenate kinase regulation of the intracellular concentration of coenzyme A. J. Biol. Chem. 275:1377-1383. https://doi. org/10.1074/jbc.275.2.1377.

Romualdi, C., S. Bortoluzzi, F. D'Alessi, and G. A. Danieli. 2003. IDEG6: A web tool for detection of differentially expressed genes in multiple tag sampling experiments. Physiol. Genomics 12:159 162.

Shi, D. S., J. Wang, Y. Yang, F. H. Lu, X. P. Li, and Q. Y. Liu. 2012. DGAT1, GH, GHR, PRL and PRLR polymorphism in water buffalo (Bubalus bubalis). Reprod. Domest. Anim. 47:328-334. https://doi.org/10.1111/j.1439-0531.2011.01876.x.

Vasudevan, S., Y. Tong, and J. A. Steitz. 2007. Switching from repression to activation: MicroRNAs can up-regulate translation. Science 318:1931-1934. https://doi.org/10.1126/science.1149460.

Wang, M., S. Moisá, M. J. Khan, J. Wang, D. Bu, and J. J. Loor. 2012 MicroRNA expression patterns in the bovine mammary gland are affected by stage of lactation. J. Dairy Sci. 95:6529-6535. https:// doi.org/10.3168/jds.2012-5748.

Watson, C. J., and T. G. Burdon. 1996. Prolactin signal transduction mechanisms in the mammary gland: The role of the Jak/Stat pathway. Rev. Reprod. 1:1-5. https://doi.org/10.1530/ror.0.0010001.

Xie, S. S., X. Y. Li, T. Liu, J. H. Cao, Q. Zhong, and S. H. Zhao. 2011. Discovery of porcine microRNAs in multiple tissues by a Solexa deep sequencing approach. PLoS One 6:e16235 https://doi. org/10.1371/journal.pone.0016235. 